\title{
Decoherence and linear entropy increase in the quantum baker's
}

\author{
map \\ Andrei N. Soklakov and Rüdiger Schack \\ Department of Mathematics, Royal Holloway, University of London, \\ Egham, Surrey TW20 0EX, UK
}

13 July 2001

\begin{abstract}
We show that the coarse-grained quantum baker's map exhibits a linear entropy increase at an asymptotic rate given by the Kolmogorov-Sinai entropy of the classical chaotic baker's map. The starting point of our analysis is a symbolic representation of the map on a string of $N$ qubits, i.e., an $N$-bit register of a quantum computer. To coarse-grain the quantum evolution, we make use of the decoherent histories formalism. As a byproduct, we show that the condition of medium decoherence holds asymptotically for the coarse-grained quantum baker's map.
\end{abstract}

The Kolmogorov-Sinai (KS) entropy of a classical dynamical system [1] quantifies the asymptotic rate at which information about the initial conditions needs to be supplied in order to retain the ability to predict the time-evolved system state with a fixed accuracy. It can also be viewed as the asymptotic linear rate of entropy increase of the coarse-grained evolution of the dynamical system. A positive KS entropy is one of the simplest and most general criteria for classical chaos. Several generalizations of KS entropy to quantum mechanics have been proposed as criteria for quantum chaos. Refs. [2, 3, 4, 5] focus on linear entropy increase, whereas Refs. [6, 7, 8] generalize the notion of unpredictability, inherent in the concept of KS entropy, to quantum mechanics.

The dynamics of an isolated quantum system is unitary and therefore entropy preserving. The entropy can grow only if there is a source of unpredictability such as coarse graining, measurement, or interaction with a heat bath [9]. The same is true classically, where for example the entropy of a coarse-grained probability distribution increases under chaotic time evolution, even though the Liouville equation preserves the entropy of the exact, fine-grained distribution. Measurement as a source of unpredictability was used in the definition of quantum dynamical entropy [5], which has been conjectured to approach KS entropy in the classical limit [5, 10, 11]. A linear growth of entropy for an inverted quantum harmonic oscillator coupled to a heat bath has been established in Ref. [3]. Most results in this field are obtained numerically (see, e.g., [12, 13, 14, 15]). In this paper we derive rigorous results using coarse graining as a source of unpredictability.

A systematic way to coarse-grain unitary quantum dynamics is provided by the decoherent histories formalism 16, 17, 18, 19. In this formalism, the quantum analogue of a coarse-graining of classical phase space takes the form of a coarse-grained history. The entropy of coarse-grained histories has been defined and analyzed in [18, 21, 20]. In this paper we give a rigorous proof that the entropy of the coarse-grained quantum baker's map exhibits an asymptotic linear growth of 
1 bit per iteration, which equals the KS entropy of the classical baker's map. In order to prove this result, we first establish that the coarse-grained histories satisfy the condition of asymptotic medium decoherence [18]. Although the consistent histories approach has been used before for the investigation of quantum dissipative chaos [22], to our knowledge this is the first time that the decoherence condition for histories has been rigorously established for a chaotic quantum system.

The quantum baker's map [23, 24] is a prototypical quantum map invented for the theoretical investigation of quantum chaos. During the last decade, it has been studied extensively (see, e.g., 25] and references therein). In this paper we consider a class of quantum baker's maps defined in [26]. These maps admit a symbolic description in terms of shifts on strings of qubits (two-state systems) similar to classical symbolic dynamics [1]. They can also be derived from the semiquantum maps introduced in [27]. See Ref. [28] for an application of symbolic methods to more general maps. The formulation and proof of the theorems below is based on the development of the symbolic description of the quantum baker's map given in [25, 29].

Quantum baker's maps are defined on the $D$-dimensional Hilbert space of the quantized unit square [30]. For consistency of units, we let the quantum scale on "phase space" be $2 \pi \hbar=1 / D$. Following Ref. [24], we choose half-integer eigenvalues $q_{j}=\left(j+\frac{1}{2}\right) / D, j=0, \ldots, D-1$, and $p_{k}=\left(k+\frac{1}{2}\right) / D, k=0, \ldots, D-1$, of the discrete "position" and "momentum" operators $\hat{q}$ and $\hat{p}$, respectively, corresponding to antiperiodic boundary conditions. We further assume that $D=2^{N}$, which is the dimension of the Hilbert space of $N$ qubits.

The $D=2^{N}$ dimensional Hilbert space modeling the unit square can be identified with the product space of $N$ qubits via

$$
\left|q_{j}\right\rangle=\left|\xi_{1}\right\rangle \otimes\left|\xi_{2}\right\rangle \otimes \cdots \otimes\left|\xi_{N}\right\rangle
$$

where $j=\sum_{l=1}^{N} \xi_{l} 2^{N-l}, \xi_{l} \in\{0,1\}$, and where each qubit has basis states $|0\rangle$ and $|1\rangle$. We can write $q_{j}$ as a binary fraction, $q_{j}=0 . \xi_{1} \xi_{2} \ldots \xi_{N} 1$. We define the notation

$$
\left|. \xi_{1} \xi_{2} \ldots \xi_{N}\right\rangle=e^{i \pi / 2}\left|q_{j}\right\rangle
$$

see Ref. [26] for the reason for the phase factor $e^{i \pi / 2}$. Momentum and position eigenstates are related through the quantum Fourier transform operator $\hat{F}$ [24], i.e., $\hat{F}\left|q_{k}\right\rangle=\left|p_{k}\right\rangle$.

By applying the Fourier transform operator to the $n$ rightmost bits of the position eigenstate $\left|\cdot \xi_{n+1} \ldots \xi_{N} \xi_{n} \ldots \xi_{1}\right\rangle$, one obtains the family of states [26]

$$
\begin{aligned}
\left|\xi_{1} \ldots \xi_{n} \cdot \xi_{n+1} \ldots \xi_{N}\right\rangle \equiv & 2^{-n / 2} e^{i \pi\left(0 . \xi_{n} \ldots \xi_{1} 1\right)}\left|\xi_{n+1}\right\rangle \otimes \cdots \otimes\left|\xi_{N}\right\rangle \otimes \\
& \left(|0\rangle+e^{2 \pi i\left(0 . \xi_{1} 1\right)}|1\rangle\right) \otimes \\
& \left(|0\rangle+e^{2 \pi i\left(0 . \xi_{2} \xi_{1} 1\right)}|1\rangle\right) \otimes \cdots \otimes \\
& \left(|0\rangle+e^{2 \pi i\left(0 . \xi_{n} \ldots \xi_{1} 1\right)}|1\rangle\right)
\end{aligned}
$$

where $1 \leq n \leq N-1$. For given $n$, these states form an orthonormal basis. The state (3) is localized in both position and momentum: it is strictly localized within a position region of width $1 / 2^{N-n}$, centered at position $q=0 . \xi_{n+1} \ldots \xi_{N} 1$, and it is crudely localized within a momentum region of width $1 / 2^{n}$, centered at momentum $p=0 . \xi_{n} \ldots \xi_{1} 1$.

For each $n, 0 \leq n \leq N-1$, a quantum baker's map can be defined by

$$
\hat{B}\left|\xi_{1} \ldots \xi_{n} \cdot \xi_{n+1} \ldots \xi_{N}\right\rangle=\left|\xi_{1} \ldots \xi_{n+1} \cdot \xi_{n+2} \ldots \xi_{N}\right\rangle
$$


where the dot is shifted by one position. In phase-space language, the map $\hat{B}$ takes a state localized at $(q, p)=\left(0 . \xi_{n+1} \ldots \xi_{N} 1,0 . \xi_{n} \ldots \xi_{1} 1\right)$ to a state localized at $\left(q^{\prime}, p^{\prime}\right)=\left(0 . \xi_{n+2} \ldots \xi_{N} 1,0 . \xi_{n+1} \ldots \xi_{1} 1\right)$, while it stretches the state by a factor of two in the $q$ direction and squeezes it by a factor of two in the $p$ direction. For $n=N-1$, the map is the original quantum baker's map as defined in Ref. [24].

We are now in a position to introduce coarse-grained sets of histories. Let us first simplify our notation slightly. Given the dimensions $N$ and $n$, the dot in the definition (3) is redundant. Thus, we will write from now on

$$
\left|\xi_{1} \ldots \xi_{N}\right\rangle \equiv\left|\xi_{1} \ldots \xi_{n} \cdot \xi_{n+1} \ldots \xi_{N}\right\rangle
$$

always keeping in mind the given values of $N$ and $n$. We introduce a set of projection operators,

$$
P_{\boldsymbol{y}}^{(l, r)} \equiv \sum_{\substack{a_{1}, \ldots, a_{l} \\ b_{1}, \ldots, b_{r}}}\left|a_{1} \ldots a_{l} \boldsymbol{y} b_{1} \ldots b_{r}\right\rangle\left\langle a_{1} \ldots a_{l} \boldsymbol{y} b_{1} \ldots b_{r}\right|,
$$

where the bold variable $\boldsymbol{y}$ denotes the binary string $\boldsymbol{y}=y_{1} \ldots y_{N-l-r}$. The operator $P_{\boldsymbol{y}}^{(l, r)}$ is a projector on a $2^{l+r}$-dimensional subspace labeled by the string $\boldsymbol{y}$. The $2^{N-l-r}$ projectors defined by all possible bit strings $\boldsymbol{y}$ form a complete set of mutually orthogonal projectors, i.e., $P_{\boldsymbol{y}}^{(l, r)} P_{\boldsymbol{y}^{\prime}}^{(l, r)}=0$ if $\boldsymbol{y} \neq \boldsymbol{y}^{\prime}$ and $\sum_{\boldsymbol{y}} P_{\boldsymbol{y}}^{(l, r)}=\mathbb{1}$. We can write each $P_{\boldsymbol{y}}^{(l, r)}$ as a diagram

$$
P_{\boldsymbol{y}}^{(l, r)} \equiv(\underbrace{\square \square \ldots \square}_{l} \boldsymbol{y} \underbrace{\square \square \ldots \square}_{r})
$$

where the empty boxes indicate $l$ leftmost and $r$ rightmost bits which are coarse-grained over. For simplicity, we will always assume in the following that $l<n$ and $r<N-n$. In this case $l$ and $r$ acquire a more specific meaning as the number of "momentum" and "position" bits ignored in the coarse-graining.

For a given dynamics, a string of projectors defines a coarse-grained history. We define two types of histories, $h_{\overrightarrow{\boldsymbol{y}}}$ and $h_{\boldsymbol{y}}^{c}$. The history $h_{\overrightarrow{\boldsymbol{y}}}$ is defined as

$$
\begin{aligned}
& h_{\overrightarrow{\boldsymbol{y}}} \equiv\left(P_{\boldsymbol{y}^{1}}^{(l, r)}, P_{\boldsymbol{y}^{2}}^{(l, r)}, \ldots, P_{\boldsymbol{y}^{k}}^{(l, r)}\right) \\
& =(\underbrace{\square \square \ldots \square}_{l} \boldsymbol{y}^{1} \underbrace{\square \square \ldots \square}_{r}, \\
& \underbrace{\square \square \ldots \square}_{l} \boldsymbol{y}^{2} \underbrace{\square \square \ldots \square}_{r}, \ldots \text {, } \\
& \underbrace{\square \square \ldots \square}_{l} \boldsymbol{y}^{k} \underbrace{\square \square \ldots \square}_{r}) \text {, }
\end{aligned}
$$

where $\overrightarrow{\boldsymbol{y}}=\left(\boldsymbol{y}^{1}, \ldots, \boldsymbol{y}^{k}\right)$. Since for each $t=1, \ldots, k$, the projectors $P_{\boldsymbol{y}^{t}}^{(l, r)}$ form a complete set of mutually orthogonal projectors, the histories $\left\{h_{\overrightarrow{\boldsymbol{y}}}\right\}$ are said to form an exhaustive set of mutually exclusive histories. They are a special case of the more general sets of histories introduced in Refs. 16, 17, 18].

The second type of histories considered here is defined by a further coarse-graining of the histories $\left\{h_{\overrightarrow{\boldsymbol{y}}}\right\}$, consisting of a summation over the first $k-1$ projectors in (8):

$$
h_{\boldsymbol{y}}^{c} \equiv(\underbrace{\mathbb{1}, \ldots, \mathbb{1}}_{k-1 \text { times }}, P_{\boldsymbol{y}}^{(l, r)}) .
$$


The histories $\left\{h_{\boldsymbol{y}}^{c}\right\}$ also form an exhaustive set of mutually exclusive histories.

Starting from some initial state $\rho_{0}$, the coarse-grained evolution of the quantum baker's map $\hat{B}$ is characterized by a decoherence functional. For the histories $\left\{h_{\overrightarrow{\boldsymbol{y}}}\right\}$, the decoherence functional is given by

$$
\mathcal{D}\left[\rho_{0}, h_{\overrightarrow{\boldsymbol{y}}}, h_{\overrightarrow{\boldsymbol{z}}}\right]=\operatorname{Tr}\left[P_{\boldsymbol{y}^{k}}^{(l, r)} \hat{B} P_{\boldsymbol{y}^{k-1}}^{(l, r)} \hat{B} \cdots P_{\boldsymbol{y}^{1}}^{(l, r)} \hat{B} \rho_{0} \hat{B}^{\dagger} P_{\boldsymbol{z}^{1}}^{(l, r)} \cdots \hat{B}^{\dagger} P_{\boldsymbol{z}^{k-1}}^{(l, r)} \hat{B}^{\dagger} P_{\boldsymbol{z}^{k}}^{(l, r)}\right],
$$

and for the histories $\left\{h_{\boldsymbol{y}}^{c}\right\}$, by

$$
\mathcal{D}\left[\rho_{0}, h_{\boldsymbol{y}}^{c}, h_{\boldsymbol{z}}^{c}\right]=\operatorname{Tr}\left[P_{\boldsymbol{y}}^{(l, r)} \hat{B}^{k} \rho_{0}\left(\hat{B}^{\dagger}\right)^{k} P_{\boldsymbol{z}}^{(l, r)}\right] .
$$

In both cases, the number of iterations of the map, $k$, is assumed to satisfy the inequality $k<r$. In the following we assume that the initial state is proportional to one of the projectors defined in Eq. (6), i.e.,

$$
\begin{aligned}
\rho_{0}=\rho_{\boldsymbol{x}}^{(l, r)} & \equiv 2^{-(l+r)} P_{\boldsymbol{x}}^{(l, r)} \\
& =2^{-(l+r)}(\underbrace{\square \square \ldots \square}_{l} \boldsymbol{x} \underbrace{\square \square \ldots \square}_{r}) .
\end{aligned}
$$

If the off-diagonal elements of the decoherence functional vanish, the set of histories is said to be decoherent (more precisely, this is the condition of medium decoherence [18]). It follows directly from the cyclic property of the trace that the coarse decoherence functional (11) satisfies the decoherence condition:

$$
\mathcal{D}\left[\rho_{0}, h_{\boldsymbol{y}}^{c}, h_{\boldsymbol{z}}^{c}\right]=0 \text { if } \boldsymbol{y} \neq \boldsymbol{z}
$$

For its diagonal elements, we have

Theorem 1: Fix two strings $\boldsymbol{x}$ and $\boldsymbol{y}$ of the same length: $|\boldsymbol{x}|=|\boldsymbol{y}|=c$. For any two strings $\boldsymbol{\alpha}$ and $\boldsymbol{\beta}$ such that $|\boldsymbol{\alpha}|=|\boldsymbol{\beta}|=k$, where $k$ is a fixed number of iterations, $k<r$, we have

$$
\mathcal{D}\left[\rho_{\boldsymbol{\alpha} \boldsymbol{x}}^{(l, r)}, h_{\boldsymbol{y} \boldsymbol{\beta}}^{c}, h_{\boldsymbol{y} \boldsymbol{\beta}}^{c}\right]=2^{-k} \delta_{\boldsymbol{x}}^{\boldsymbol{y}}-O\left(\frac{l+r}{2^{l-k}}\right),
$$

where $\boldsymbol{\alpha} \boldsymbol{x}$ denotes concatenation of the strings $\boldsymbol{\alpha}$ and $\boldsymbol{x}$ and similarly for $\boldsymbol{y} \boldsymbol{\beta}$, and where $\delta_{\boldsymbol{x}}^{\boldsymbol{y}}$ denotes the Kronecker delta function. The proof of this and the results below will be given in a subsequent longer paper.

Since the decoherence condition is satisfied, we can interpret the diagonal elements (14) as probabilities. We see that there is no single dominant history. Instead, after the $k$-th step there are $2^{k}$ different histories each having asymptotically the same probability, $2^{-k}$. These histories are defined by the condition $\boldsymbol{x}=\boldsymbol{y}$, i.e., a shift of $k$ binary positions to the left:

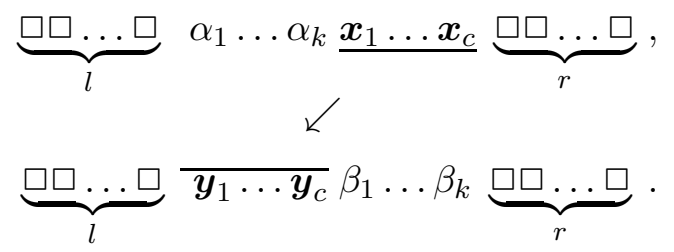

During this transformation the bits of $\boldsymbol{\alpha}$ are lost as they reach the scale at which the momentum becomes coarse-grained. At the same time $k$ unspecified (i.e., random) position bits $\beta_{1} \ldots \beta_{k}$ enter 
the relevant section of the string. At each step the number of histories with significant probability doubles, as each history branches into two equiprobable histories. This means there is a loss of one bit of information per iteration.

We now give a precise formulation of this information loss. Since the set of histories $\left\{h_{\boldsymbol{y}}^{c}\right\}$ is decoherent, we can define its entropy [20, 21, 18],

$$
H\left(\left\{h_{\boldsymbol{y}}^{c}\right\}\right) \equiv-\sum_{\boldsymbol{y}} p\left(h_{\boldsymbol{y}}^{c}\right) \log _{2} p\left(h_{\boldsymbol{y}}^{c}\right),
$$

where $p\left(h_{\boldsymbol{y}}^{c}\right)=\mathcal{D}\left[\rho_{\boldsymbol{x}}^{(l, r)}, h_{\boldsymbol{y}}^{c}, h_{\boldsymbol{y}}^{c}\right]$. Using theorem 1 , we find that

$$
H\left(\left\{h_{\boldsymbol{y}}^{c}\right\}\right)=k+O\left(\frac{(l+r) \log _{2}(l+r)}{2^{l-k}}\right) .
$$

The results for the very coarse histories $h_{\boldsymbol{y}}^{c}$ depend in part on the fact that the decoherence condition is trivially satisfied for these histories. In the more interesting case of the less coarse-grained histories $\left\{h_{\overrightarrow{\boldsymbol{y}}}\right\}$, the decoherence condition is satisfied only asymptotically. The following theorem establishes this asymptotic decoherence and gives asymptotic values for the diagonal elements of the decoherence functional.

Theorem 2: Fix any integer $\gamma \geq 1$, any string $\boldsymbol{x}$ of length $|\boldsymbol{x}|=\gamma$, and any two ordered sequences of strings $\overrightarrow{\boldsymbol{y}}=\left(\boldsymbol{y}^{1}, \boldsymbol{y}^{2}, \ldots, \boldsymbol{y}^{k}\right)$ and $\overrightarrow{\boldsymbol{z}}=\left(\boldsymbol{z}^{1}, \boldsymbol{z}^{2}, \ldots, \boldsymbol{z}^{k}\right)$ such that $\left|\boldsymbol{y}^{j}\right|=\left|\boldsymbol{z}^{j}\right|=\gamma, j=1, \ldots, k$, where $k$ is the number of iterations, $k<r$. For sufficiently large $l$ we have then:

$$
\begin{aligned}
& \mathcal{D}\left[\rho_{\boldsymbol{x}}^{(l, r)}, h_{\overrightarrow{\boldsymbol{y}}}, h_{\overrightarrow{\boldsymbol{z}}}\right]=2^{-k}\left(\prod_{j=1}^{k} \delta_{\boldsymbol{y}^{j}}^{\boldsymbol{z}^{j}}\right)\left(\prod_{j=1}^{k-1} \delta_{\boldsymbol{y}_{1: \gamma-1}^{j+1}}^{\boldsymbol{y}_{2: \gamma}^{j}} \delta_{\boldsymbol{y}_{1}^{j}}^{\boldsymbol{x}_{j+1}}\right) \delta_{\boldsymbol{y}_{1: \gamma-k}^{k}}^{\boldsymbol{x}_{k+1: \gamma}}+O\left(\frac{l+r-k}{2^{l-2\left(k^{2}+k\right)}}\right)
\end{aligned}
$$

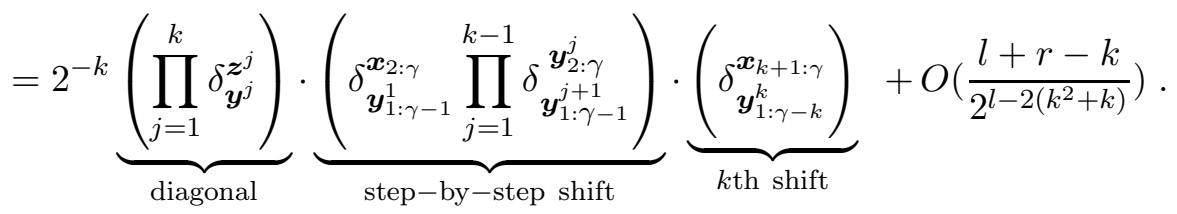

The second equality provides a somewhat redundant but more transparent formulation of the theorem.

We see that the expression in the first parentheses is zero for all off-diagonal elements of the decoherence functional. This implies that in the limit of large $l$ all off-diagonal elements of the decoherence functional vanish, which establishes the medium decoherence condition. The diagonal elements of the decoherence functional can therefore be interpreted as probabilities of the corresponding histories (see Ref. [19] for a discussion of approximate decoherence). Asymptotically, only $2^{k}$ diagonal elements are nonzero. Moreover, the error terms are exponentially small. As in the case of the coarse histories considered above, there are $2^{k}$ histories with asymptotically equal probabilities. The number of such histories doubles after each step resulting in a loss of information at the rate of 1 bit per step. The conditions satisfied by the histories with nonzero probabilities are also similar to the previous case. Here, each of these histories is a sequence of $k$ projectors and each of those projectors is related to the initial state via a shift according to the position of the projector in the history:

$$
\underbrace{\square \square \ldots \square}_{l} \boldsymbol{x}_{1} \underline{\boldsymbol{x}_{2} \ldots \boldsymbol{x}_{\gamma-2} \boldsymbol{x}_{\gamma-1} \boldsymbol{x}_{\gamma}} \underbrace{\square \square \ldots \square}_{r},
$$




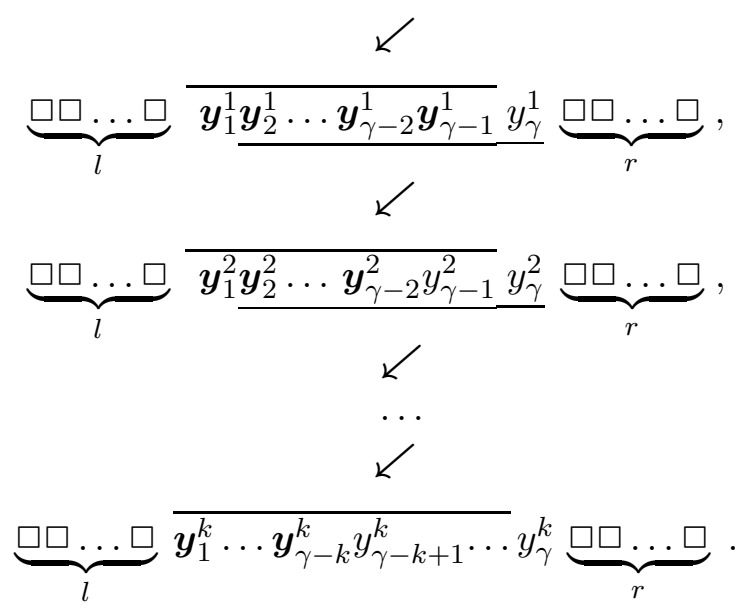

In this diagram the first line represents the initial condition $\rho_{\boldsymbol{x}}^{(l, r)}$. The subsequent lines correspond to the projectors $P_{\boldsymbol{y}^{1}}^{(l, r)}, \ldots, P_{\boldsymbol{y}^{k}}^{(l, r)}$ in the history. The bold face is used to indicate the bits which are completely determined by the initial condition for those histories with asymptotically nonzero probability. Such histories satisfy the step-by-step shift condition denoted on the diagram by the arrows and lines: for example, the substring $\boldsymbol{x}_{2} \ldots \boldsymbol{x}_{\gamma}$ is shifted onto the substring $\boldsymbol{y}_{1}^{1} \ldots \boldsymbol{y}_{\gamma-1}^{1}$. For the entire history, therefore, there are only $k$ independent bits which can be chosen arbitrarily, given the step-by-step shift constraint. We recover the coarse-histories case considered above if we choose $y_{\gamma-k+1}^{k} \ldots y_{\gamma}^{k}$ as independent and record only the very last projector, ignoring the rest of the trajectory.

The entropy of the approximately decoherent set of histories $\left\{h_{\overrightarrow{\boldsymbol{y}}}\right\}$ is

$$
H\left(\left\{h_{\overrightarrow{\boldsymbol{y}}}\right\}\right)=-\sum_{\overrightarrow{\boldsymbol{y}}} p\left(h_{\overrightarrow{\boldsymbol{y}}}\right) \log _{2} p\left(h_{\overrightarrow{\boldsymbol{y}}}\right)
$$

where $p\left(h_{\overrightarrow{\boldsymbol{y}}}\right)=\mathcal{D}\left[\rho_{\boldsymbol{x}}^{(l, r)}, h_{\overrightarrow{\boldsymbol{y}}}, h_{\overrightarrow{\boldsymbol{y}}}\right]$. It follows then from theorem 2 that

$$
H\left(\left\{h_{\overrightarrow{\boldsymbol{y}}}\right\}\right)=k+O\left(\frac{(l+r-k) \log _{2}(l+r-k)}{2^{l-2\left(k^{2}+k\right)}}\right) .
$$

In the limit of large $l$, for any fixed number of iterations, $k$, the entropy of the coarse-grained quantum baker's map approaches the value of $k$ bits, i.e., 1 bit per iteration, which is the KS entropy of the classical baker's map. Due to the $k^{2}$ term in the denominator, the bound on the error term is not as tight as in Eq. (17). We believe that this bound can be further improved.

\section{References}

[1] V. M. Alekseev and M. V. Yakobson, Phys. Reports 75, 287 (1981).

[2] P. Pechukas, J. Phys. Chem. 86, 2239 (1982).

[3] W. H. Zurek and J. P. Paz, Phys. Rev. Lett. 72, 2508 (1994).

[4] R. Alicki and M. Fannes, Lett. Math. Phys. 32, 75 (1994). 
[5] W. Słomczyński and K. Życzkowski, J. Math. Phys. 35, 5674 (1994).

[6] R. Schack and C. M. Caves, Phys. Rev. Lett. 71, 525 (1993).

[7] R. Schack and C. M. Caves, Phys. Rev. E 53, 3257 (1996).

[8] R. Schack and C. M. Caves, Phys. Rev. E 53, 3387 (1996).

[9] C. M. Caves and R. Schack, Complexity 3, 46 (1997).

[10] W. Słomczyński and K. Życzkowski, Phys. Rev. Lett. 80, 1880 (1998).

[11] P. Pakoński, A. Ostruszka, and K. Życzkowski, Nonlinearity 12, 269 (1999).

[12] R. Alicki, D. Makowiec, and W. Miklaszewski, Phys. Rev. Lett. 77, 838 (1996).

[13] J. Kwapien, W. Słomczyński, and K. Życzkowski, J. Phys. A 30, 3175 (1997).

[14] S. Habib, K. Shizume, and W. H. Zurek, Phys. Rev. Lett. 80, 4361 (1998).

[15] P. A. Miller and S. Sarkar, Nonlinearity 12, 419 (1999).

[16] R. Griffiths, J. Stat. Phys. 36, 219 (1984).

[17] R. Omnès, J. Stat. Phys. 53, 893, 933, 957 (1988).

[18] M. Gell-Mann and J. B. Hartle, in Complexity, Entropy, and the Physics of Information, edited by W. H. Zurek (Addison Wesley, Redwood City, CA, 1990).

[19] H. F. Dowker and J. J. Halliwell, Phys. Rev. D 46, 1580 (1992).

[20] T. A. Brun and J. B. Hartle, Phys. Rev. E 59, 6370 (1999).

[21] J. B. Hartle, Physica Scripta T76, 67 (1998).

[22] T. A. Brun, Phys. Lett. A 206, 167 (1995).

[23] N. L. Balazs and A. Voros, Ann. Phys. 190, 1 (1989).

[24] M. Saraceno, Ann. Phys. 199, 37 (1990).

[25] A. N. Soklakov and R. Schack, Phys. Rev. E 61, 5108 (2000).

[26] R. Schack and C. M. Caves, Applicable Algebra in Engineering, Communication and Computing (AAECC) 10, 305 (2000).

[27] M. Saraceno and A. Voros, Physica D 79, 206 (1994).

[28] R. O. Vallejos and M. Saraceno, J. Phys. A 32, 7273 (1999).

[29] A. N. Soklakov, Measures of dynamical complexity, PhD thesis (Royal Holloway, University of London, 2001), also available as technical report RHUL-MA-2001-1, http://www.rhul.ac.uk/mathematics/techreports.

[30] H. Weyl, The Theory of Groups and Quantum Mechanics (Dover, New York, 1950). 